\title{
Depth of response is a significant predictor for long-term outcome in advanced gastric cancer patients treated with trastuzumab
}

\author{
Choong-Kun Lee ${ }^{1}$, Seung-Seob Kim², Saemi Park ${ }^{3}$, Chan Kim ${ }^{1}$, Su Jin Heo ${ }^{1}$, Joon \\ Seok Lim ${ }^{2}$, Hyunki Kim ${ }^{4}$, Hyo Song Kim ${ }^{1}$, Sun Young Rha ${ }^{1}$, Hyun Cheol Chung ${ }^{1}$, \\ Sohee Park ${ }^{3}$, Minkyu Jung ${ }^{1}$ \\ ${ }^{1}$ Division of Medical Oncology, Department of Internal Medicine, Yonsei Cancer Center, Yonsei University, College of Medicine, \\ Seoul, Korea \\ ${ }^{2}$ Department of Radiology, Yonsei University College of Medicine, Seoul, Korea \\ ${ }^{3}$ Department of Biostatistics, Graduate School of Public Health, Yonsei University, Seoul, Korea \\ ${ }^{4}$ Department of Pathology, Yonsei University College of Medicine, Seoul, Korea \\ Correspondence to: Sohee Park, email: soheepark@yuhs.ac \\ Minkyu Jung, email: minkjung@yuhs.ac
}

Keywords: gastric cancer, trastuzumab, depth of response, early tumor shrinkage, survival

Received: December 14, 2016

Accepted: March 01, 2017

Published: March 10, 2017

Copyright: Lee et al. This is an open-access article distributed under the terms of the Creative Commons Attribution License (CC-BY), which permits unrestricted use, distribution, and reproduction in any medium, provided the original author and source are credited.

\section{ABSTRACT}

Purpose: We aimed to determine and compare the predictive values of depth of response (DpR) and early tumor shrinkage (ETS) on long-term outcomes in gastric cancer patients treated with trastuzumab.

Results: From a total of 368 computed tomography examinations, DpR and ETS were evaluated. DpR was a significant tumor-size metric in predicting PFS and OS, and showed better discriminatory ability (higher $C_{T}$ indices, 0.6957 for PFS; 0.7191 for OS) than ETS. DpR $\geq 45 \%$ (vs. < 45\%) was the optimal cutoff value, as it was best able to identify patients with longer PFS (median 9.0 vs. 6.3 months, hazard ratio $[H R]=0.608 ; 95 \%$ confidence interval $[\mathrm{CI}]: 0.335$ to $1.104 ; P=0.102$ ) and $0 S$ (median 23.5 vs. 13.1 months, $\mathrm{HR}=0.441 ; 95 \% \mathrm{CI}: 0.203$ to $0.955 ; P=0.038$ ).

Materials and Methods: Sixty-one gastric cancer patients who received first-line trastuzumab-based chemotherapy were assessed for DPR and ETS. We employed Kaplan-Meier estimates, log-rank tests, Cox proportional hazards regression models, time-dependent receiver operating characteristics, and Youden's $J$ index to evaluate and determine cutoff values of DpR and ETS as predictors of progression-free survival (PFS) and overall survival (OS).

Conclusions: DPR and ETS were significant predictors of long-term outcomes in gastric cancer patients treated with first-line trastuzumab. Validation in prospective trials with larger patient populations is needed.

\section{INTRODUCTION}

Gastric cancer, the second leading cause of cancerrelated deaths worldwide, remains a major health problem despite a global decrease in its incidence [1]. Currently, trastuzumab-based first-line treatments represent the standard approach for human epidermal growth factor receptor 2 (HER2)-positive advanced gastric cancer (AGC) patients [2]; however, it is not equally effective in all such patients. Although identifying patients who will most benefit from this treatment is paramount, clinically applicable surrogate markers for survival have not been properly evaluated in AGC patients treated with trastuzumab. Even as $H E R 2$ gene amplification was reported to predict overall survival (OS) in AGC patients undergoing trastuzumabbased chemotherapy [3], HER2 gene amplification is not usually tested in patients who receive a score of $3+$ on HER2 immunohistochemistry (IHC).

Defining appropriate surrogate end-points that predict long-term survival in prospective studies is important in the field of oncology. Aside from well-known surrogate end-points for OS, such as progression-free 
survival (PFS) and objective response rate, other metrics based on tumor size have recently been investigated. These include early tumor shrinkage (ETS) and depth of response (DpR), both of which are indicators of the percentage change in tumor size at a designated time point from the baseline. ETS, defined as change in tumor size at the time of first response evaluation, has been proposed to be associated with long-term outcomes in various cancer types and regimens, mainly colorectal cancer [4-10]. Recently, the concept of DpR [11], defined as the maximal tumor shrinkage observed [12], has gained attention in colorectal cancer patients treated with cetuximab or bevacizumab. Increased DpR was observed to significantly correlate with post-progression survival (PPS) or OS in cetuximab- or bevacizumab-treated colorectal cancer patients in large phase III trials $[11,13,14]$. Analysis of tumor-size metrics in association with survival has never been performed for gastric cancer. HER2-positive gastric cancers, which usually metastasize to measurable lesions in lymph nodes or the liver $[3,15]$ are an ideal system in which to test the usage of DpR or ETS as surrogate markers for long-term outcome. Additionally, while there have been separate studies on the association of DpR or ETS and cancer patient prognosis, there has been no study evaluating DpR or ETS as predictive factors for survival outcome within one treatment cohort, particularly in gastric cancer patients.

The purpose of this study was to investigate the predictive values of DpR and ETS on long-term outcomes in AGC patients treated with trastuzumab-based chemotherapy. We also sought to perform our investigation by using tumorsize metrics as surrogate markers and defining discriminatory cutoff values for dichotomizing patient populations according to DpR or ETS to guide treatment planning.

\section{RESULTS}

\section{Patients}

A total of 61 of 88 gastric cancer patients treated with trastuzumab were eligible for this study (Supplementary Figure 1). Patients underwent trastuzumab treatment between December 2005 and March 2014, with a median follow-up duration of 24.3 months (interquartile range, 18.3 to 28.3 months). The main clinicopathological features and response data are shown in Table 1. HER2/CEP17 ratio by SISH or FISH was categorized into two groups using a cutoff of 4.7 , a previously reported cutoff value that predicted response and survival [3]. The median number of trastuzumab cycles administered was eight (range, 2 to 38).

\section{Comparing DpR with ETS in predicting long- term outcomes}

Relative tumor sizes compared to the baseline (the sum of the longest diameters according to RECIST) at the first evaluation (ETS) and at the best response (DpR) were evaluated from a total of 368 CT examinations (see Figure 1 for schematic illustration of tumor size metrics). The median time between the first trastuzumab administration and the first response evaluation (time to ETS) was 6 weeks (interquartile range, 5.3 to 7.1 weeks). In a univariate analysis, Eastern Cooperative Oncology Group (ECOG) performance status and HER2 overexpression as determined by IHC were significantly related to PFS and OS. Among the tumor size metrics, DpR was most strongly associated with both PFS and OS $(P<0.001)$, followed by time to tumor growth (TTG) and ETS (Supplementary Table 1). A multivariable Cox proportional hazards model for patient prognosis, adjusted for potential confounding factors including age, ECOG performance status, and HER2 overexpression, showed that DpR and ETS were significant predictors of long-term outcomes of both PFS and OS (Table 2 and Supplementary Table 2).

\section{Comparing the predictive accuracies of $\mathrm{DpR}$ and ETS as continuous variables for long-term outcomes}

Time-dependent receiver operating characteristics (TDROC) analysis was performed to compare the predictive accuracy of DpR and ETS as continuous variables for long-term outcomes (Table 3). DpR showed better discriminatory ability (higher $\mathrm{C}_{\tau}$ indices, 0.6957 [95\% CI: 0.6564 to 0.8176] for PFS; 0.7191 [95\% CI: 0.6694 to 0.8465$]$ for OS) than ETS $\left(\mathrm{C}_{\tau}\right.$ indices, 0.6722 [95\% CI: 0.6131 to 0.7807$]$ for PFS; 0.6681 [95\% CI: 0.6040 to 0.7952 ] for $\mathrm{OS})$. Higher $\mathrm{C}_{\tau}$ indices were found for DpR than for ETS consistently for both PFS and OS over time (Figure 2). Time-specific accuracy decreased minimally over time for OS with both DpR and ETS (Figure 2D) yet appeared consistent in the TDROC curves of DpR and PFS (Figure 2B).

\section{Clinical applicability of DpR and ETS}

\section{Choice of cutoff values}

The clinical utility of tumor-size metrics often involves dichotomization of the population around a cutoff threshold that best distinguishes the long-term outcomes between groups. In this analysis, we used Youden's $J$ index, a commonly used measure of overall diagnostic effectiveness, to obtain the optimal cutoff points for both DpR and ETS. ROC curves of DpR and ETS depicting Youden's $J$ index and optimal cutoff points for PFS or OS were constructed (Supplementary Figure 2). Cutoff values of $29.3 \%$ (PFS) and $27.7 \%$ (OS) for ETS and $44.4 \%$ (PFS) and $43.3 \%$ (OS) for DpR were obtained based on the highest sensitivity and specificity (Table 3). Cutoff values of $30 \%$ for ETS and $45 \%$ for DpR were chosen for further analyses. 


\begin{tabular}{|c|c|c|}
\hline Characteristics & $\mathbf{N}$ & $\%$ \\
\hline \multicolumn{3}{|l|}{ Age } \\
\hline Median & \multicolumn{2}{|c|}{60} \\
\hline Range & \multicolumn{2}{|c|}{$33-80$} \\
\hline \multicolumn{3}{|l|}{ Sex } \\
\hline Male & 40 & 65.6 \\
\hline Female & 21 & 34.4 \\
\hline \multicolumn{3}{|l|}{ ECOG performance status } \\
\hline 0 & 31 & 50.8 \\
\hline 1 & 22 & 36.1 \\
\hline 2 & 8 & 13.1 \\
\hline \multicolumn{3}{|l|}{ WHO classification } \\
\hline Adenocarcinoma well differentiated & 5 & 8.2 \\
\hline Adenocarcinoma moderately differentiated & 37 & 60.7 \\
\hline Adenocarcinoma poorly differentiated & 14 & 23 \\
\hline Signet ring cell & 5 & 8.2 \\
\hline \multicolumn{3}{|l|}{ Prior gastrectomy } \\
\hline Subtotal gastrectomy & 8 & 13.1 \\
\hline Total gastrectomy & 7 & 11.5 \\
\hline Not done & 46 & 75.4 \\
\hline \multicolumn{3}{|l|}{ Primary tumor location } \\
\hline Gastroesophageal junction & 4 & 6.6 \\
\hline Cardia & 10 & 16.4 \\
\hline Body/Antrum & 47 & 77 \\
\hline \multicolumn{3}{|l|}{ HER2 overexpression by IHC } \\
\hline $2+$ & 15 & 24.6 \\
\hline $3+$ & 46 & 75.4 \\
\hline \multicolumn{3}{|l|}{ HER2/CEP17 ratio by SISH or FISH } \\
\hline $2.0-4.69$ & 11 & 18.0 \\
\hline$\geq 4.7$ & 11 & 18.0 \\
\hline Not determined & 39 & 63.9 \\
\hline \multicolumn{3}{|l|}{ Metastatic location } \\
\hline Lymph node & 47 & 77.0 \\
\hline Liver & 27 & 44.3 \\
\hline Peritoneum* & 17 & 27.9 \\
\hline Lung & 8 & 13.1 \\
\hline Bone & 5 & 8.2 \\
\hline \multicolumn{3}{|l|}{ Chemotherapy regimen } \\
\hline Herceptin + FP & 9 & 14.8 \\
\hline Herceptin + XP & 52 & 85.2 \\
\hline \multicolumn{3}{|l|}{ Follow-up, months } \\
\hline Median & \multicolumn{2}{|c|}{24.3} \\
\hline Interquartile range & \multicolumn{2}{|c|}{$18.3-28.3$} \\
\hline
\end{tabular}




\section{Progression-free survival, months}

Events (Progression or Death)

54

Median

$95 \% \mathrm{CI}$

Overall survival, months

Events (Death)

Median

$95 \% \mathrm{CI}$
8.0

$6.8-9.3$

35

16.0

$11.2-21.0$

Abbreviations: CI, confidence interval; ECOG, Eastern Cooperative Oncology Group; WHO, World Health Organization; HER2, human epidermal growth factor receptor 2; IHC, immunohistochemistry; CEP17, chromosome 17 centromere; SISH, silver in situ hybridization; FISH, fluoroscence in situ hybridization; FP, 5-FU+Cisplatin; XP, Xeloda+Cisplatin. * 11 patients had measurable peritoneal seeding nodules.

\section{Long-term outcome prediction using DpR and ETS}

Patients were divided into subgroups according to cutoff values for ETS $(<30 \%: N=23$; $\geq 30 \%: n=$ 38) or $\operatorname{DpR}(<45 \%: N=24$; $\geq 45 \%: N=37)$, and their clinicopathological features and survivals were compared (Supplementary Table 3). Both groups had similar characteristics except for a better performance status and higher HER2 overexpression on IHC among the better response subgroups. PFS and OS were significantly longer for patients in the $\geq 45 \% \mathrm{DpR}$ group yet not for those in the $\geq 30 \%$ ETS group. Kaplan-Meier plots for PFS and OS in relation to DpR and ETS, each categorized by their respective cutoff values, were also constructed (Figure 3). Dichotomized DpR was a significant predictor of PFS $(P=0.007)$ and OS $(P=0.006)$, although ETS was not $(P=0.076$ for PFS; $P=0.109$ for OS). Using a multivariable Cox proportional hazards model adjusted for potentially confounding prognostic factors such as age, ECOG performance status, and HER2 overexpression, it was found that the cutoff value of $\mathrm{DpR} \geq 45 \%$ (vs. $<45 \%$ ) was a significant predictor for OS (median 23.5 vs. 13.1 months, hazard ratio $[\mathrm{HR}]=0.441 ; 95 \% \mathrm{CI}=0.204$ to 0.955; Table 4).

\section{Post-progression survival prediction using DpR and ETS}

Predicting survival after progression is potentially useful for selecting patients recommended for secondline chemotherapy or further clinical trials. For this purpose, a subgroup of patients who received secondline chemotherapy $(N=37)$ were evaluated for PPS and its relation to DpR or ETS. On univariate analysis, changes in DpR were significantly correlated with PPS (Supplementary Table 4). After adjusting for age, ECOG performance status, and HER2 overexpression using IHC, DpR was the only independent predictive factor for PPS $(\mathrm{HR}=0.844 ; 95 \% \mathrm{CI}=0.712$ to 0.999 ; Supplementary Table 5). The cutoff value of $45 \%$ was

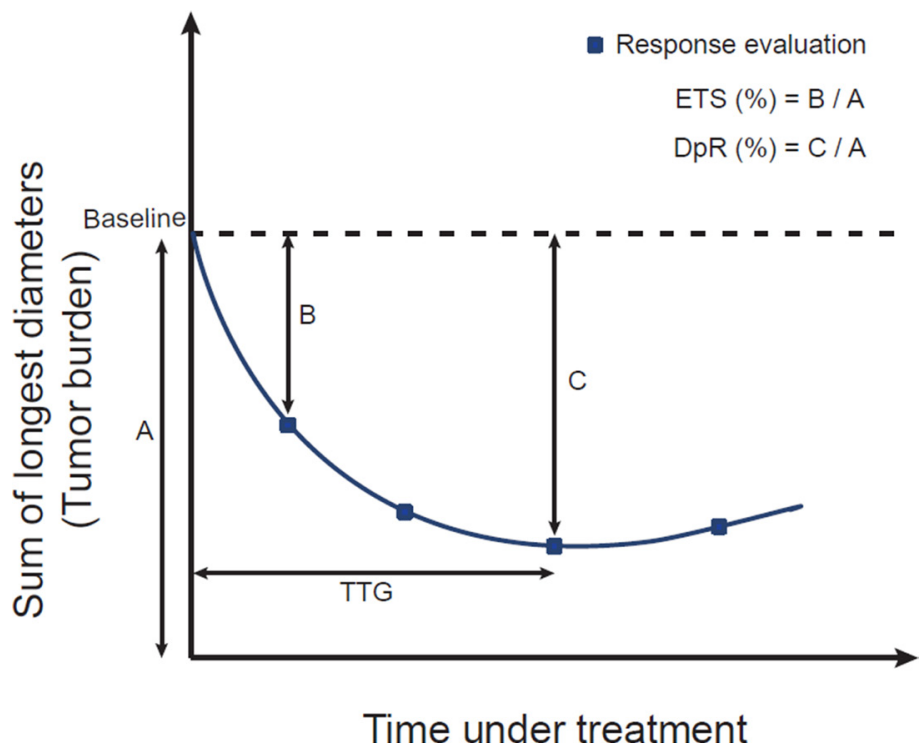

Figure 1: Schematic illustration for metrics of tumor size. ETS, early tumor shrinkage; DpR, depth of response; TTG, time to tumor growth. 
Table 2: Multivariable analysis of selected demographic variables

\begin{tabular}{|c|c|c|c|c|c|c|c|}
\hline & \multicolumn{3}{|c|}{ Progression-free Survival } & \multicolumn{3}{|c|}{ Overall Survival } \\
\hline & & \multicolumn{2}{|c|}{ Adjusted for Covariates } & \multicolumn{4}{|c|}{ Adjusted for Covariates } \\
\hline & & HR & $95 \% \mathrm{CI}$ & $P$ & HR & $95 \% \mathrm{CI}$ & $\boldsymbol{P}$ \\
\hline \multirow[t]{2}{*}{ Age } & $<65$ & Ref & & & Ref & & \\
\hline & $\geq 65$ & 0.777 & 0.423 to 1.426 & 0.415 & 1.001 & 0.482 to 2.069 & 0.997 \\
\hline \multirow[t]{2}{*}{ ECOG performance status } & 0 & Ref & & & Ref & & \\
\hline & $\geq 1$ & 1.746 & 0.967 to 3.153 & 0.065 & 2.284 & 1.090 to 4.785 & 0.029 \\
\hline \multirow[t]{2}{*}{ HER2 overexpression by IHC } & $2+$ & Ref & & & Ref & & \\
\hline & $3+$ & 0.529 & 0.282 to 0.993 & 0.048 & 1.092 & 0.486 to 2.453 & 0.832 \\
\hline $\mathrm{ETS}^{\dagger}$ & & 0.858 & 0.765 to 0.961 & 0.008 & 0.869 & 0.758 to 0.996 & 0.044 \\
\hline \multirow[t]{2}{*}{ Age } & $<65$ & Ref & & & Ref & & \\
\hline & $\geq 65$ & 0.796 & 0.441 to 1.436 & 0.448 & 1.119 & 0.536 to 2.335 & 0.764 \\
\hline \multirow[t]{2}{*}{ ECOG performance status } & 0 & Ref & & & Ref & & \\
\hline & $\geq 1$ & 1.415 & 0.775 to 2.582 & 0.258 & 1.876 & 0.868 to 4.055 & 0.110 \\
\hline \multirow[t]{2}{*}{ HER2 overexpression by IHC } & $2+$ & Ref & & & Ref & & \\
\hline & $3+$ & 0.626 & 0.333 to 1.179 & 0.147 & 1.616 & 0.671 to 3.891 & 0.284 \\
\hline $\mathrm{DpR}^{\dagger}$ & & 0.786 & 0.700 to 0.883 & $<0.0001$ & 0.798 & 0.698 to 0.912 & 0.001 \\
\hline
\end{tabular}

$\dagger$ HR per $10 \%$ tumor size decrease

Abbreviations: HR, hazard ratio; CI, confidence interval; Ref, Reference; ECOG, Eastern Cooperative Oncology Group; HER2, human epidermal growth factor receptor 2; IHC, immunohistochemistry; ETS, early tumor shringkage; TTG, time to tumor growth; DpR, depth of response.

a significant predictor of patients with longer PPS $(P=0.02$; Figure 3F $)$.

\section{DISCUSSION}

To our knowledge, our study is among the first to evaluate $\mathrm{DpR}$ as a predictor of long-term outcome and to compare the predictive abilities of DpR and ETS in AGC patients. Both DpR and ETS were significant predictors for PFS and OS in AGC patients who were treated with first-line trastuzumab-containing chemotherapy.
We recognized DpR as a novel and effective measure of treatment outcome and conducted our study to compare DpR with ETS in order to determine which tumor-size metric is more predictive of prognosis. In recent large randomized phase III trials comparing cetuximab in combination with FOLFIRI as first-line treatments in colorectal cancer patients [13], the two arms did not show a difference in PFS or objective response rate, although the OS was longer in the FOLFIRI-plus-cetuximab arm. An independent radiological review suggested that the increased DpR observed in the FOLFIRI-plus-cetuximab
A

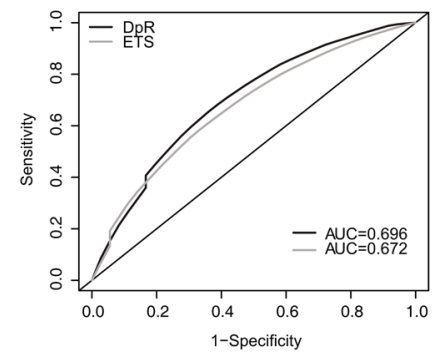

B

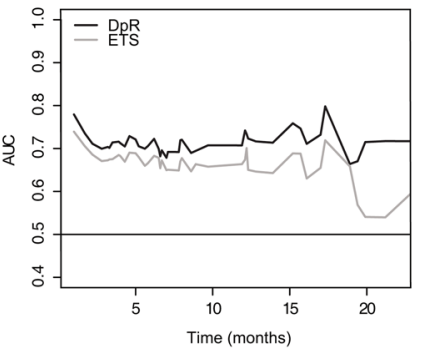

C

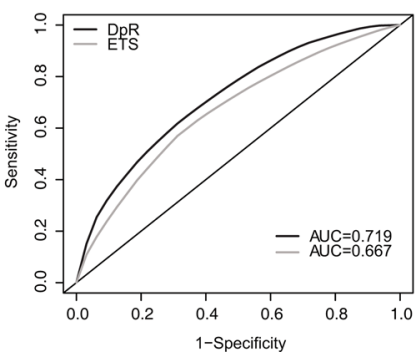

$\mathrm{D}$

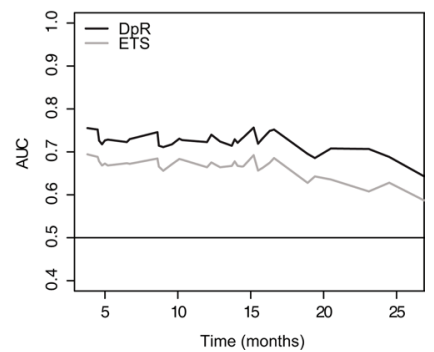

Figure 2: Time-dependent receiver operating characteristics curves of DpR and ETS for progression-free survival at 6.7 months* (A) and overall survival at 13.8 months* $(\mathbf{C})$; time-dependent integrated AUC of DpR and ETS for progression-free survival (B) and overall survival (D). *6.7 months and 13.8 months were chosen from median progression-free survival and median overall survival data reported for the Trastuzumab for Gastric Cancer (ToGA) trial. AUC, area under the curve; ETS, early tumor shrinkage; DpR, depth of response. 
Table 3: Performance indices of early tumor shrinkage and depth of response on long-term outcome

\begin{tabular}{llcc}
\hline & & Progression-free Survival & Overall Survival \\
\hline ETS & & & \\
& C $\tau$ index & 0.6722 & 0.6681 \\
& $95 \% \mathrm{CI}^{\dagger}$ & 0.6131 to 0.7807 & 0.6040 to 0.7952 \\
& Youden Index & 0.2516 & 0.2573 \\
& Cutoff Value & $29.3 \%$ & $27.7 \%$ \\
DpR & & 0.6957 & 0.7191 \\
& $\mathrm{C} \tau$ index & 0.6564 to 0.8176 & 0.6694 to 0.8465 \\
& $95 \% \mathrm{CI}^{\dagger}$ & 0.2943 & 0.3056 \\
& Youden Index & $44.4 \%$ & $43.3 \%$ \\
\hline
\end{tabular}

$\dagger 95 \%$ CI was obtained from bootstrapping of 1000 samples.

Abbreviations: ETS, early tumor shrinkage; $\mathrm{DpR}$, depth of response; $\mathrm{C} \tau$, time-dependent concordance; $\mathrm{CI}$, confidence interval.

arm may explain in part the significant OS advantage [16]. This result implies that responses to therapy might not be adequately evaluated when performed by only using RECIST; however, RECIST-independent tumorsize metrics such as DpR can better predict or categorize patients who will respond to therapy or survive longer. As DpR or ETS can easily be calculated from numbers obtained through RECIST measurements, DpR can swiftly be adopted in future clinical trials as another surrogate marker or a secondary end-point and can consequently be adopted routinely in clinical practice.

By employing Youden's $J$ index, we found the optimal cutoff values to be $30 \%$ for ETS and $45 \%$ for DpR; however, only the DpR cutoff successfully classified patients according to their survival prognosis. Our identification of $\mathrm{DpR}$ as a dichotomous variable (45\% cutoff point) makes it easily applicable to clinical practice. When adjusted for potential confounding factors, it was found that the cutoff value of $\mathrm{DpR} \geq 45 \%(v s .<45 \%)$ was a significant predictor for OS yet not for PFS (Table 4). This result also supports the notion of using DpR as a surrogate marker for OS.

The survival benefit of second-line chemotherapy compared to best supportive care has been demonstrated in several recent studies in patients with AGC [17-21]. Although second-line treatment now represents a standard of care in AGC, predictive factors regarding the efficacy of second-line chemotherapy are needed, especially considering the limited benefit of available therapies. Therefore, we examined whether DpR or ETS can predict PPS, as that could potentially serve as a guide for designing second-line chemotherapy or clinical trials. DpR was the only relevant factor in this case; a cutoff value of $\geq 45 \%$ identified patients with better PPS. Therefore, studies examining the efficacy of second-line therapies could be stratified according to the DpR achieved during first-line therapy. Our results of gastric cancer patients on first-line Trastuzumab with $\mathrm{DpR} \geq 45 \%$ showing longer PPS and OS imply that a certain subgroup of patients with partial response (response rate $\geq 30 \%$ ) yet without major DpR change (45\%) will eventually have worse prognosis in terms of PPS or OS. For this group of patients, considering the bad prognosis of the advanced gastric cancer patients with short PPS, clinicians might consider a change to second-line chemotherapy for patients on first-line chemotherapy without improvement of cancer-related symptoms. Furthermore, the importance of DpR in predicting PPS was reported in KRAS wildtype colorectal cancer patients treated with cetuximabbased chemotherapy [11], implying that this notion can be applied to other tumor types and anti-tumor agents.

As a previous study revealed that TTG was one of the metrics that predicted OS in colorectal cancer patients receiving cetuximab [12], we re-conducted a multivariable analysis with DpR, including TTG as a covariate, and confirmed that DpR was a significant prognostic factor for survival outcome (Supplementary Table 6). One confounding factor was the absolute tumor burden. However, when multivariable analyses included the adjusted baseline sum of the longest diameters of target lesions (tumor burden) as a factor, DpR nevertheless remained significantly associated with OS and PFS in our study (Supplementary Table 7).

While this study revealed several valuable findings, it also had several limitations or arguments. First, this study was a retrospective study by design; thus, there may have been potential confounding factors in predicting long-term survival outcomes. Second, this study had a relatively small sample size; however, the patients included in this study were very homogeneous, which helped to minimize variability in patient prognoses. Third, as the patients in this study were treated with both trastuzumab and cytotoxic chemotherapy (XP or FP), we could not conclude that our results, which demonstrated the ability of DpR and ETS to predict prognostic outcome in HER2-positive gastric cancer patients, were 
A

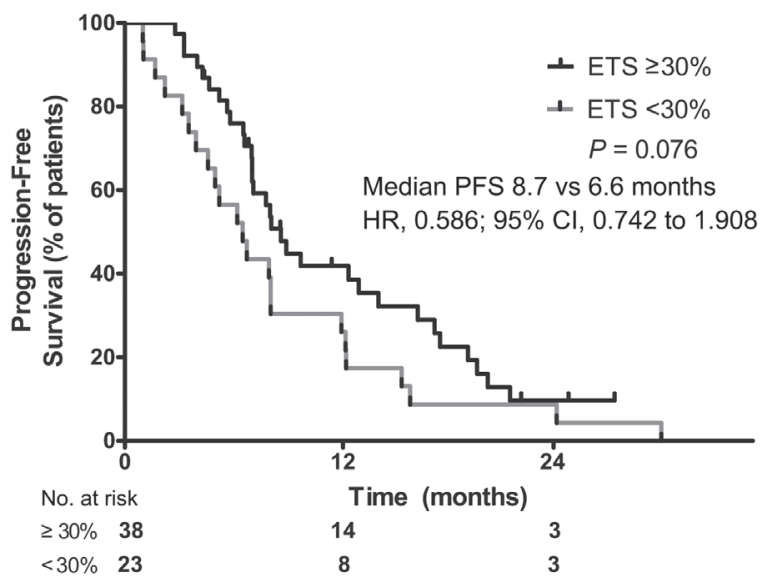

C

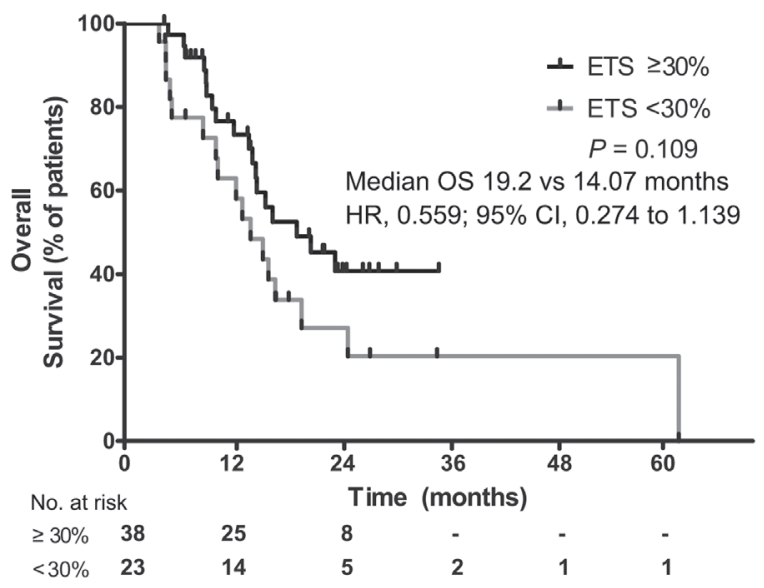

$E$

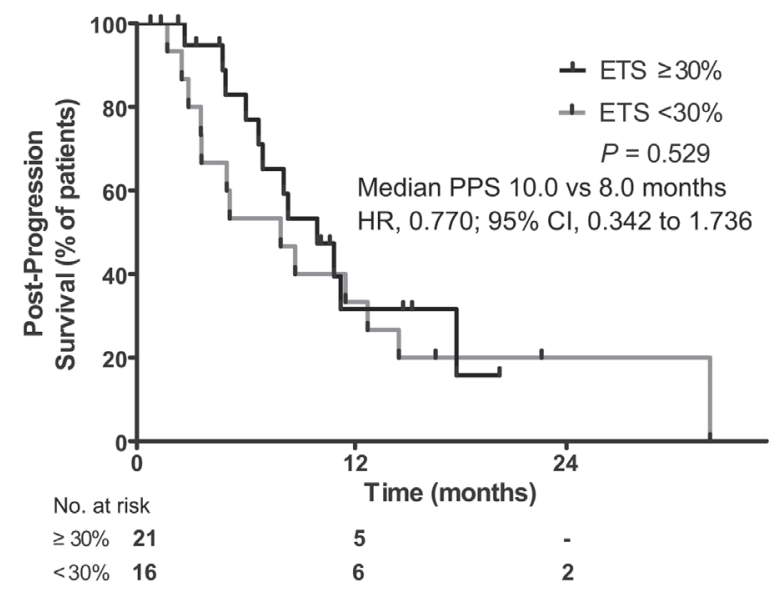

B
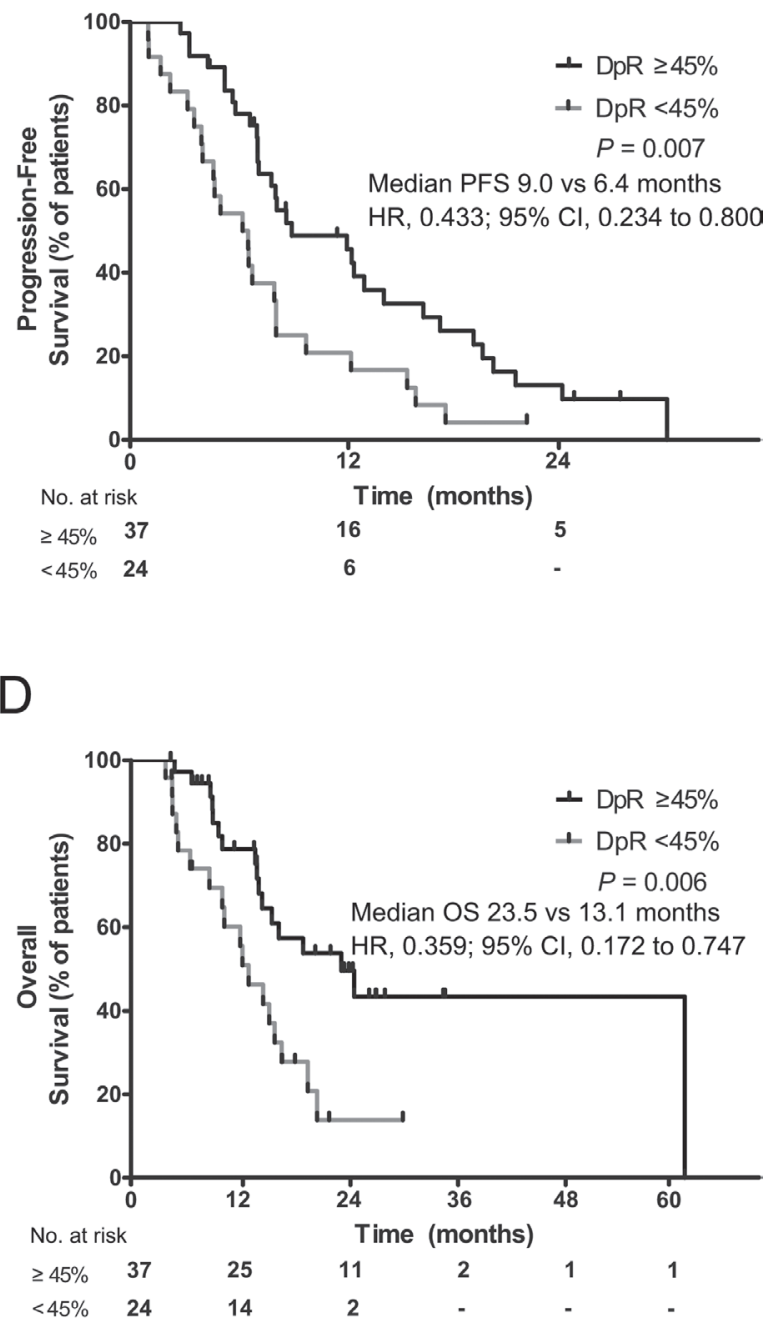

$\mathrm{F}$

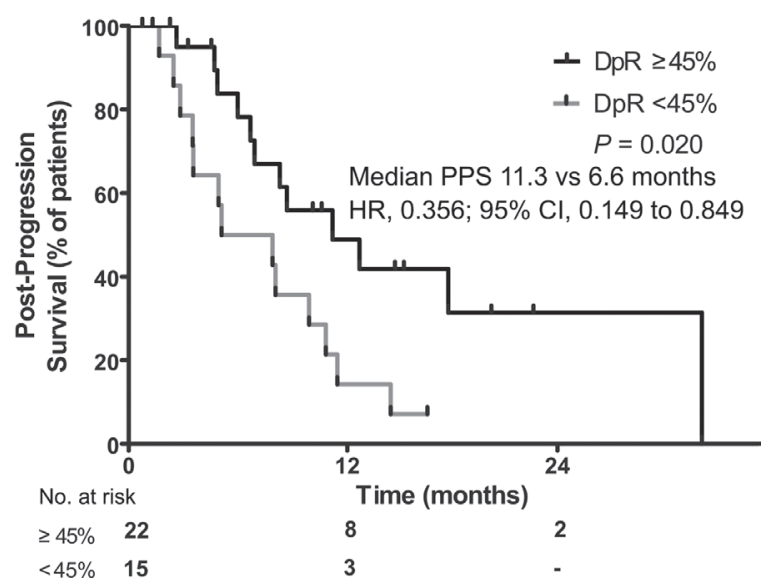

Figure 3: Kaplan-Meier curves for progression-free survival $(\mathbf{A}, \mathbf{B})$, overall survival $(\mathbf{C}, \mathbf{D})$, and post-progression survival $(\mathbf{E}, \mathbf{F})$ in relation to early tumor shrinkage (cutoff $\geq 30 \%$; A, C, E) and depth of response (cutoff $\geq 45 \%$; B, D, F). ETS, early tumor shrinkage; DpR, depth of response. 
Table 4: Multivariable analysis of selected demographic variables and ETS or DpR categorized by cutoff values

\begin{tabular}{|c|c|c|c|c|c|c|c|}
\hline & & \multicolumn{3}{|c|}{ Progression-free Survival } & \multicolumn{3}{|c|}{ Overall Survival } \\
\hline & & \multicolumn{2}{|c|}{ Adjusted for Covariates } & \multirow[b]{2}{*}{$\boldsymbol{P}$} & \multicolumn{2}{|c|}{ Adjusted for Covariates } & \multirow[b]{2}{*}{$\boldsymbol{P}$} \\
\hline & & HR & $95 \% \mathrm{CI}$ & & HR & $95 \% \mathrm{CI}$ & \\
\hline \multirow[t]{2}{*}{ Age } & $<65$ & Ref & & & Ref & & \\
\hline & $\geq 65$ & 0.816 & 0.433 to 1.540 & 0.531 & 0.945 & 0.441 to 2.026 & 0.885 \\
\hline \multirow[t]{2}{*}{ ECOG performance status } & 0 & Ref & & & Ref & & \\
\hline & $\geq 1$ & 1.689 & 0.928 to 3.075 & 0.086 & 2.404 & 1.145 to 5.049 & 0.021 \\
\hline \multirow[t]{2}{*}{ HER 2 overexpression by IHC } & $2+$ & Ref & & & Ref & & \\
\hline & $3+$ & 0.521 & 0.274 to 0.993 & 0.048 & 0.949 & 0.429 to 2.100 & 0.897 \\
\hline \multirow[t]{2}{*}{ ETS } & $<30 \%$ & Ref & & & Ref & & \\
\hline & $\geq 30 \%$ & 0.665 & 0.371 to 1.193 & 0.171 & 0.687 & 0.332 to 1.423 & 0.312 \\
\hline \multirow[t]{2}{*}{ Age } & $<65$ & Ref & & & Ref & & \\
\hline & $\geq 65$ & 0.896 & 0.488 to 1.626 & 0.720 & 1.025 & 0.488 to 2.147 & 0.950 \\
\hline \multirow[t]{2}{*}{ ECOG performance status } & 0 & Ref & & & Ref & & \\
\hline & $\geq 1$ & 1.582 & 0.861 to 2.915 & 0.141 & 2.213 & 1.045 to 4.675 & 0.038 \\
\hline \multirow[t]{2}{*}{ HER 2 overexpression by IHC } & $2+$ & Ref & & & Ref & & \\
\hline & $3+$ & 0.587 & 0.298 to 1.827 & 0.122 & 1.224 & 0.526 to 2.848 & 0.639 \\
\hline \multirow[t]{2}{*}{ DpR } & $<45 \%$ & Ref & & & Ref & & \\
\hline & $\geq 45 \%$ & 0.609 & 0.335 to 1.107 & 0.104 & 0.441 & 0.204 to 0.955 & 0.038 \\
\hline
\end{tabular}

Abbreviations: HR, hazard ratio; CI, confidence interval; Ref, Reference; ECOG, Eastern Cooperative Oncology Group; HER2, human epidermal growth factor receptor 2; IHC, immunohistochemistry; ETS, early tumor shrinkage; DpR, depth of response

attributable to the effect of trastuzumab alone. However, the proportion of patients who scored $3+$ for HER2 overexpression by IHC was higher among patients with ETS $\geq 30 \%$ and $\mathrm{DpR} \geq 45 \%$ than that of those who scored $2+(P=0.042$ and $P=0.003$, respectively; Supplementary Table 3), suggesting that DpR and ETS are good metrics for patients treated with trastuzumab. Additionally, there was no statistically significant difference between patients who received adjuvant chemotherapy after curative gastrectomy $(n=9)$ and chemo-naive patients $(n=52)$ in terms of PFS (Supplementary Figure 4). This result also supports the hypothesis that cytotoxic chemotherapy does not have a great effect on the ability of DpR and ETS to predict prognostic outcome in HER2-positive gastric cancer patients. Even if the results were attributable to cytotoxic chemotherapy, this study nevertheless highlights the importance of $\mathrm{DpR}$ in predicting long-term outcomes. Fourth, DpR or ETS were mainly evaluated from measurable metastatic sites, some might argue that the HER2 tests were mainly performed in primary cancer tissues and therefore DpR or ETS may not fully reflect the antitumor effect of Herceptin. However, since current clinical practice or guidelines do not have any recommendation for re-assessment of HER2 status from metastatic sites, and since previous studies reported high concordance rates of HER 2 status between primary gastric cancer and paired metastatic sites [22, 23], we think that DpR or ETS would reflect anti-tumor effect of Herceptin from primary sites. Lastly, as biologically gastric cancer in the East differs from that in the West, doubts could be raised regarding the applicability of this study's results to gastric cancer patients in the West. Western countries have a much higher incidence of gastric cancer that is located in the gastroesophageal junction, and there is a higher prevalence of diffuse histology in Western gastric cancer patients [24]. HER2-positivity rates are higher in specimens from the gastroesophageal junction than in specimens from the body of the stomach and are also higher in the histologically intestinal type than in mixed or diffuse types [25]. Less than $10 \%$ of the patients in this study had gastroesophageal-junction cancer; thus, we could not generally adopt our concept of DpR and ETS in predicting long-term outcomes in gastric cancer patients treated with trastuzumab to patients in the West. However, considering that there were no differences in HER2positivity between European and Asian countries based on 
the ToGA trial screening data ( $23.9 \%$ vs. $23.6 \% ; n=3665)$ and the Western patients more significantly responded to trastuzumab than the Eastern patients in the ToGA trial, we expect that DpR and ETS will be significant predictors of long-term outcomes among Western gastric cancer patients treated with trastuzumab as well.

In conclusion, our results strongly suggest that $\mathrm{DpR}$ is a significant predictor of long-term outcomes including PFS, OS, and PPS among AGC patients treated with trastuzumab. We suggest a cutoff value of $\mathrm{DpR} \geq 45 \%$ to identify patients with better prognoses; this could guide clinical decision making and further identify patients likely to obtain additional benefits from trastuzumab or further chemotherapies. Moreover, DpR may be a promising and valuable endpoint in designing future clinical trials. Further validation of these results in prospective trials with larger populations and with other tumor types is warranted.

\section{MATERIALS AND METHODS}

\section{Patients and assessments}

A single-institution retrospective cohort analysis was performed at Yonsei Cancer Center, Seoul, Korea. The study included HER2-positive locally advanced, recurrent, or metastatic gastric cancer patients who were histologically confirmed to be inoperable and had been treated with first-line capecitabine plus cisplatin (XP) or fluorouracil plus cisplatin (FP) combined with trastuzumab. Patients with progression or death events before the first scheduled response evaluation time were excluded from the study cohort and used in the landmark analysis. Patients without measurable lesions as defined by the Response Evaluation Criteria in Solid Tumors (RECIST) version 1.1 [26] were also excluded. Electronic medical records were analyzed retrospectively in accordance with the Declaration of Helsinki. The Institutional Review Board in Severance Hospital, Seoul, Korea, reviewed and approved this study (IRB approval Number: 4-2014-1076).

Tumor samples were deemed HER2-positive if their HER2 scores were $3+$ on IHC or if they stained positive for HER2 on silver in situ hybridization (SISH) or fluorescence in situ hybridization (FISH; HER2:CEP17 ratio $\geq 2$ ). Two independent radiologists blinded to patient data assessed target lesion responses at various time points using computed tomography (CT) according to RECIST version 1.1 every two cycles until disease progression or withdrawal. The relative change in the sum of the longest diameters of target lesions at the first response evaluation compared to baseline was defined as ETS. DpR was defined as the relative change in the sum of the target lesions' longest diameters at their smallest attained sizes compared to baseline (TTG; time to tumor growth). PFS was defined as the interval between the first trastuzumab administration and the first documentation of progression or death; OS was calculated as the time between first trastuzumab administration and death of any cause. PPS was defined as the survival difference between OS and PFS (PPS $=$ OS - PFS).

\section{Statistical analysis}

Survival probabilities were estimated using the Kaplan-Meier method, and a log-rank test was used to evaluate differences between survival curves. A Cox proportional hazards regression model was used for univariate and multivariable analyses. Multivariable timedependent concordance $\left(\mathrm{C}_{\tau}\right)$ indices and area under the curve (AUC) values for long-term outcomes were obtained from time-dependent receiver operating characteristics (TDROC) curve analyses to evaluate sensitivity and specificity [27]. Nonparametric bootstrapping with 1000 replicates was used to determine the $95 \%$ confidence intervals (CIs) of the $\mathrm{C}_{\tau}$ indices. Youden's $J$ index was calculated to determine the threshold point at which to dichotomize the patient population based on $\mathrm{DpR}$ or ETS in order to predict the PFS or OS [28]. All $P$-values were two-sided, and $P<0.05$ was considered statistically significant.

SPSS version 20 was used for survival analysis via the Kaplan-Meier method. The statistical software package $\mathrm{R}$ version 13.0 was used to run all other analyses including TDROC and the Cox proportional hazards regression model.

\section{ACKNOWLEDGMENTS AND FUNDING}

This work was supported by the Yonsei University Future-leading Research Initiative of 2015 (2015-22-0159).

\section{CONFLICTS OF INTEREST}

The authors indicated no financial relationships.

\section{REFERENCES}

1. Jemal A, Bray F, Center MM, Ferlay J, Ward E, Forman D. Global cancer statistics. CA Cancer J Clin. 2011; 61:69-90.

2. Bang YJ, Van Cutsem E, Feyereislova A, Chung HC, Shen L, Sawaki A, Lordick F, Ohtsu A, Omuro Y, Satoh T, Aprile G, Kulikov E, Hill J, et al. Trastuzumab in combination with chemotherapy versus chemotherapy alone for treatment of HER2-positive advanced gastric or gastrooesophageal junction cancer (ToGA): a phase 3, open-label, randomised controlled trial. Lancet. 2010; 376:687-697.

3. Gomez-Martin C, Plaza JC, Pazo-Cid R, Salud A, Pons F, Fonseca P, Leon A, Alsina M, Visa L, Rivera F, Galan MC, Del Valle E, Vilardell F, et al. Level of HER2 gene amplification predicts response and overall survival 
in HER2-positive advanced gastric cancer treated with trastuzumab. J Clin Oncol. 2013; 31:4445-4452.

4. Seidel C, Busch J, Weikert S, Steffens S, Bokemeyer C, Grunwald V. Tumour shrinkage measured with first treatment evaluation under VEGF-targeted therapy as prognostic marker in metastatic renal cell carcinoma (mRCC). Br J Cancer. 2013; 109:2998-3004.

5. Piessevaux H, Buyse M, Schlichting M, Van Cutsem E, Bokemeyer C, Heeger S, Tejpar S. Use of early tumor shrinkage to predict long-term outcome in metastatic colorectal cancer treated with cetuximab. J Clin Oncol. 2013; 31:3764-3775.

6. Modest DP, Laubender RP, Stintzing S, Giessen C, Schulz C, Haas M, Mansmann U, Heinemann V. Early tumor shrinkage in patients with metastatic colorectal cancer receiving first-line treatment with cetuximab combined with either CAPIRI or CAPOX: an analysis of the German AIO KRK 0104 trial. Acta Oncol. 2013; 52:956-962.

7. Giessen C, Laubender RP, Fischer von Weikersthal L, Schalhorn A, Modest DP, Stintzing S, Haas M, Mansmann UR, Heinemann V. Early tumor shrinkage in metastatic colorectal cancer: retrospective analysis from an irinotecan-based randomized first-line trial. Cancer Sci. 2013; 104:718-724.

8. Piessevaux H, Buyse $M$, De Roock W, Prenen $H$, Schlichting M, Van Cutsem E, Tejpar S. Radiological tumor size decrease at week 6 is a potent predictor of outcome in chemorefractory metastatic colorectal cancer treated with cetuximab (BOND trial). Ann Oncol. 2009; 20:1375-1382.

9. Claret L, Girard P, Hoff PM, Van Cutsem E, Zuideveld KP, Jorga K, Fagerberg J, Bruno R. Model-based prediction of phase III overall survival in colorectal cancer on the basis of phase II tumor dynamics. J Clin Oncol. 2009; 27:4103-4108.

10. Wang Y, Sung C, Dartois C, Ramchandani R, Booth BP, Rock E, Gobburu J. Elucidation of relationship between tumor size and survival in non-small-cell lung cancer patients can aid early decision making in clinical drug development. Clin Pharmacol Ther. 2009; 86:167-174.

11. Mansmann UR, Sartorius U, Laubender RP, Giessen CA, Esser R, Heinemann V. Deepness of response: A quantitative analysis of its impact on post-progression survival time after first-line treatment in patients with mCRC. J Clin Oncol. 2013; 31.

12. Claret L, Gupta M, Han K, Joshi A, Sarapa N, He J, Powell B, Bruno R. Evaluation of tumor-size response metrics to predict overall survival in Western and Chinese patients with first-line metastatic colorectal cancer. J Clin Oncol. 2013; 31:2110-2114.

13. Heinemann $\mathrm{V}$, von Weikersthal LF, Decker $\mathrm{T}$, Kiani A, Vehling-Kaiser U, Al-Batran SE, Heintges T, Lerchenmuller C, Kahl C, Seipelt G, Kullmann F, Stauch M, Scheithauer W, et al. FOLFIRI plus cetuximab versus FOLFIRI plus bevacizumab as first-line treatment for patients with metastatic colorectal cancer (FIRE-3): a randomised, open-label, phase 3 trial. Lancet Oncol. 2014; 15:1065-1075.

14. Cremolini C, Loupakis F, Antoniotti C, Lonardi S, Masi G, Salvatore L, Cortesi E, Tomasello G, Spadi R, Zaniboni A, Tonini G, Barone C, Vitello S, et al. Early Tumor Shrinkage and Depth of Response predict long-term outcome in metastatic colorectal cancer patients treated with first-line chemotherapy plus bevacizumab: results from phase III TRIBE trial by the Gruppo Oncologico del Nord Ovest. Ann Oncol. 2015; doi: 10.1093/annonc/mdv112.

15. Aizawa M, Nagatsuma AK, Kitada K, Kuwata T, Fujii S, Kinoshita T, Ochiai A. Evaluation of HER2-based biology in 1,006 cases of gastric cancer in a Japanese population. Gastric Cancer. 2014; 17:34-42.

16. Stintzing S, Modest DP, Weikersthal LFv, Decker T, Kiani A, Vehling-Kaiser U, Al-Batran S, Heintges T, Lerchenmueller C, Kahl C, Seipelt G, Kullmann F, Scheithauer W, et al. Independent radiological evaluation of objective response, early tumor shrinkage, and depth of response in FIRE-3 (AIO KRK-0306) in the final RAS evaluable population. Ann Oncol. 2014; 25:v11.

17. Thuss-Patience PC, Kretzschmar A, Bichev D, Deist T, Hinke A, Breithaupt K, Dogan Y, Gebauer B, Schumacher G, Reichardt P. Survival advantage for irinotecan versus best supportive care as second-line chemotherapy in gastric cancer - a randomised phase III study of the Arbeitsgemeinschaft Internistische Onkologie (AIO). Eur J Cancer. 2011; 47:2306-2314.

18. Kang JH, Lee SI, Lim do H, Park KW, Oh SY, Kwon HC, Hwang IG, Lee SC, Nam E, Shin DB, Lee J, Park JO, Park YS, et al. Salvage chemotherapy for pretreated gastric cancer: a randomized phase III trial comparing chemotherapy plus best supportive care with best supportive care alone. J Clin Oncol. 2012; 30:1513-1518.

19. Ford HE, Marshall A, Bridgewater JA, Janowitz T, Coxon FY, Wadsley J, Mansoor W, Fyfe D, Madhusudan S, Middleton GW, Swinson D, Falk S, Chau I, et al. Docetaxel versus active symptom control for refractory oesophagogastric adenocarcinoma (COUGAR-02): an open-label, phase 3 randomised controlled trial. Lancet Oncol. 2014; 15:78-86.

20. Fuchs CS, Tomasek J, Yong CJ, Dumitru F, Passalacqua R, Goswami C, Safran H, dos Santos LV, Aprile G, Ferry DR, Melichar B, Tehfe M, Topuzov E, et al. Ramucirumab monotherapy for previously treated advanced gastric or gastro-oesophageal junction adenocarcinoma (REGARD): an international, randomised, multicentre, placebocontrolled, phase 3 trial. Lancet. 2014; 383:31-39.

21. Wilke H, Muro K, Van Cutsem E, Oh SC, Bodoky G, Shimada Y, Hironaka S, Sugimoto N, Lipatov O, Kim TY, Cunningham D, Rougier P, Komatsu Y, et al. Ramucirumab plus paclitaxel versus placebo plus paclitaxel in patients with previously treated advanced gastric or gastro-oesophageal junction adenocarcinoma (RAINBOW): a double-blind, randomised phase 3 trial. Lancet Oncol. 2014; 15:1224-1235. 
22. Bozzetti C, Negri FV, Lagrasta CA, Crafa P, Bassano C, Tamagnini I, Gardini G, Nizzoli R, Leonardi F, Gasparro D, Camisa R, Cavalli S, Silini EM, et al. Comparison of HER2 status in primary and paired metastatic sites of gastric carcinoma. Br J Cancer. 2011; 104:1372-1376.

23. Kochi M, Fujii M, Masuda S, Kanamori N, Mihara Y, Funada T, Tamegai H, Watanabe M, Suda H, Takayama T. Differing deregulation of HER2 in primary gastric cancer and synchronous related metastatic lymph nodes. Diagn Pathol. 2013; 8:191.

24. Bickenbach K, Strong VE. Comparisons of Gastric Cancer Treatments: East vs. West. J Gastric Cancer. 2012; 12:55-62.

25. Van Cutsem E, Bang YJ, Feng-Yi F, Xu JM, Lee KW, Jiao SC, Chong JL, Lopez-Sanchez RI, Price T, Gladkov
O, Stoss O, Hill J, Ng V, et al. HER2 screening data from ToGA: targeting HER2 in gastric and gastroesophageal junction cancer. Gastric Cancer. 2015; 18:476-484.

26. Eisenhauer EA, Therasse P, Bogaerts J, Schwartz LH, Sargent D, Ford R, Dancey J, Arbuck S, Gwyther S, Mooney M, Rubinstein L, Shankar L, Dodd L, et al. New response evaluation criteria in solid tumours: revised RECIST guideline (version 1.1). Eur J Cancer. 2009; 45:228-247.

27. Heagerty PJ, Zheng Y. Survival model predictive accuracy and ROC curves. Biometrics. 2005; 61:92-105.

28. Youden WJ. Index for rating diagnostic tests. Cancer. 1950; $3: 32-35$. 